PROCEEDINGS OF THE

AMERICAN MATHEMATICAL SOCIETY

Volume 137, Number 4, April 2009, Pages 1255-1264

S 0002-9939(08)09700-1

Article electronically published on October 22, 2008

\title{
A CHARACTERIZATION OF FINITE PREHOMOGENEOUS VECTOR SPACES ASSOCIATED WITH PRODUCTS OF SPECIAL LINEAR GROUPS AND DYNKIN QUIVERS
}

\author{
MAKOTO NAGURA, SHIN-ICHI OTANI, AND DAISUKE TAKEDA \\ (Communicated by Martin Lorenz)
}

\begin{abstract}
For a given finite-type quiver $\Gamma$, we will consider scalar-removed representations $\left(S_{d}, R_{d}(\Gamma)\right)$, where $S_{d}$ is a direct product of special linear algebraic groups and $R_{d}(\Gamma)$ is the representation defined naturally by $\Gamma$ and a dimension vector $d$. In this paper, we give a necessary and sufficient condition on $d$ that $R_{d}(\Gamma)$ has only finitely many $S_{d}$-orbits. This condition can be paraphrased as a condition concerning lattices of small rank spanned by positive roots of $\Gamma$. To determine such scalar-removed representations having only finitely many orbits is very fundamental to the open problem of classification of the so-called semisimple finite prehomogeneous vector spaces. We consider everything over an algebraically closed field of characteristic zero.
\end{abstract}

\section{INTRODUCTION}

Let $\Gamma=\left(\Gamma_{0}, \Gamma_{1}\right)$ be a quiver with $r$ vertices (here $\Gamma_{0}$, respectively $\Gamma_{1}$, is the set of vertices, respectively arrows). Then for an $r$-tuple of non-negative integers $d=\left(d^{(i)}\right)_{i \in \Gamma_{0}}$ (we call it a dimension vector), the group $G_{d}=\prod_{i \in \Gamma_{0}} G L\left(d^{(i)}\right)$ acts naturally on $R_{d}(\Gamma)=\bigoplus_{\alpha \in \Gamma_{1}} M\left(d^{(e \alpha)}, d^{(s \alpha)}\right)$, where we consider everything over an algebraically closed field of characteristic zero, and we denote by $M\left(d^{(e \alpha)}, d^{(s \alpha)}\right)$ the vector space consisting of $d^{(e \alpha)} \times d^{(s \alpha)}$ matrices and by $s \alpha$ (resp. e $\alpha$ ) the starting (resp. ending) point for an arrow $\alpha \in \Gamma_{1}$. We will call $\left(G_{d}, R_{d}(\Gamma)\right)$ a representation associated with $\Gamma$.

In general, let $\rho: G \rightarrow G L(V)$ be a rational representation of a connected linear algebraic group $G$ on a finite-dimensional vector space $V$. If $V$ is decomposed into a finite union of $G$-orbits, it must have a unique Zariski dense orbit; hence $(G, V)$ is a prehomogeneous vector space (abbreviated PV). Such a PV is called a finite $P V$ (abbreviated FP). If $G$ is semisimple, we call $(G, V)$ semisimple. Some classes of FPs have already been classified, for example, by Sato-Kimura [9, §8] in the case of irreducible $\rho$, by Kimura-Kasai-Yasukura [6] in the case where each irreducible component has sufficient scalar multiplication, and by Kimura-Kamiyoshi-MakiOuchi-Takano [5] in the case of type $\left(G \times G L_{n}, \rho \otimes \Lambda_{1}\right)$.

In the case where $\Gamma$ is finite-type (i.e., its underlying graph is one of the Dynkin diagrams of type $A_{n}, D_{n}, E_{6}, E_{7}$, or $\left.E_{8}\right)$, it is well-known that $\left(G_{d}, R_{d}(\Gamma)\right)$ is an

Received by the editors April 22, 2008.

2000 Mathematics Subject Classification. Primary 14L30; Secondary 16G20, 11S90.

Key words and phrases. Semisimple finite prehomogeneous vector space, Dynkin quiver.

(C)2008 American Mathematical Society Reverts to public domain 28 years from publication 
FP for arbitrary $d$. However, the scalar-removed representation $\left(S_{d}, R_{d}(\Gamma)\right)$, where $S_{d}=\prod_{i \in \Gamma_{0}} S L\left(d^{(i)}\right) \subset G_{d}$, may not be a PV, and not even an FP. Note that the condition whether $\left(S_{d}, R_{d}(\Gamma)\right)$ is an FP does not depend on the choice of an orientation of $\Gamma$, but the condition whether it is a PV does. In the case where $\Gamma$ is of type $A_{n}$, it is known that such a condition can be characterized by existence of a certain relative invariant (see $\S 5$ of $[7]$ ). Such a characterization via relative invariants, however, fails even for $D_{4}$-type $\Gamma$, and it seems more complicated, in general, to write down concretely the condition whether $\left(S_{d}, R_{d}(\Gamma)\right)$ for a given dimension vector $d$ is an FP or not (see Theorem 4.1).

In this paper, we classify all scalar-removed FPs associated with finite-type quivers. According to the result of [6], to classify such scalar-removed FPs is fundamental to the classification of all semisimple (i.e., without any scalar multiplication) FPs, because $(G, \rho, V)$ is also necessarily an FP if $\left(H,\left.\rho\right|_{H}, V\right)$ for a subgroup $H \subset G$ is an FP. Our theorem (Theorem 3.4) gives a necessary and sufficient condition, for a given dimension vector $d$, whether $\left(S_{d}, R_{d}(\Gamma)\right)$ is an FP or not. As mentioned in 93 , this condition can be paraphrased as a condition whether a certain lattice of small rank spanned by positive roots of $\Gamma$ contains $d$ or not. This viewpoint gives us a lucid explanation for conditions on $d$ (look again at the twenty conditions listed in Theorem 4.1); that is, to determine FPs, $\left(S_{d}, R_{d}(\Gamma)\right)$ is nothing but determining lattices of small rank spanned by positive roots. Thus, for an arbitrary finite-type quiver $\Gamma$ and a dimension vector $d$, we can mechanically determine whether a given representation $\left(S_{d}, R_{d}(\Gamma)\right)$ is an $\mathrm{FP}$ or not.

\section{Preliminaries}

We consider everything over an algebraically closed field $\mathbb{K}$ of characteristic zero.

Let $\Gamma=\left(\Gamma_{0}, \Gamma_{1}\right)$ be a quiver with $r$ vertices, where $\Gamma_{0}=\{1,2, \ldots, r\}$ is the set of vertices and $\Gamma_{1}$ is the set of arrows. For each arrow $\alpha \in \Gamma_{1}$, we denote its starting point, respectively ending point, by $s \alpha$, respectively $e \alpha$; for example, if $i \stackrel{\alpha}{\longrightarrow} j$ for an arrow $\alpha \in \Gamma_{1}$, we have $s \alpha=i$ and $e \alpha=j$.

For an $r$-tuple of non-negative integers $\boldsymbol{d}=\left(d^{(i)}\right)_{i \in \Gamma_{0}}$ (we will call such an $r$ tuple a dimension vector), the direct product of general linear algebraic groups $G_{\boldsymbol{d}}=\prod_{i \in \Gamma_{0}} G L\left(d^{(i)}\right)$ acts on the vector space $R_{\boldsymbol{d}}(\Gamma)=\bigoplus_{\alpha \in \Gamma_{1}} M\left(d^{(e \alpha)}, d^{(s \alpha)}\right)$ by $g \cdot X=\left(g^{(e \alpha)} X^{(\alpha)}\left(g^{(s \alpha)}\right)^{-1}\right)_{\alpha \in \Gamma_{1}}$ for $g=\left(g^{(i)}\right)_{i \in \Gamma_{0}} \in G_{\boldsymbol{d}}$ and $X=\left(X^{(\alpha)}\right)_{\alpha \in \Gamma_{1}} \in$ $R_{\boldsymbol{d}}(\Gamma)$, where we denote by $M\left(d^{(i)}, d^{(j)}\right)$ the set of $d^{(i)} \times d^{(j)}$ matrices. In the case of $d^{(i)}=0$, we will consider corresponding things to be trivial. We call $\left(G_{\boldsymbol{d}}, R_{\boldsymbol{d}}(\Gamma)\right)$ a representation associated with $\Gamma$.

On the other hand, each element of the vector space $R_{\boldsymbol{d}}(\Gamma)$ is sometimes called a representation of $\Gamma$. In such a context we call $\boldsymbol{d}$ the dimension of $X \in R_{\boldsymbol{d}}(\Gamma)$ and denote it by $\operatorname{dim} X=\boldsymbol{d}$. For two representations $X$ and $Y$ of $\Gamma$ with the same dimension $\boldsymbol{d}$ (that is, $X, Y \in R_{\boldsymbol{d}}(\Gamma)$ ), we say that they are isomorphic if $X$ and $Y$ belong to the same $G_{\boldsymbol{d}^{-}}$orbit. We will express such representations as $X \cong Y$.

Let $X$ and $Y$ be representations of $\Gamma$ with dimensions $\boldsymbol{d}$ and $\boldsymbol{d}^{\prime}$, respectively. We define their direct sum $X \oplus Y$ by

$$
X \oplus Y=\left(\left[\begin{array}{cc}
X^{(\alpha)} & 0 \\
0 & Y^{(\alpha)}
\end{array}\right]\right)_{\alpha \in \Gamma_{1}} .
$$

This is a representation of $\Gamma$ with dimension $\boldsymbol{d}+\boldsymbol{d}^{\prime}$, that is, an element of the vector space $R_{\boldsymbol{d}+\boldsymbol{d}^{\prime}}(\Gamma)$. If a representation $X$ cannot be expressed as the direct 
sum of two non-zero representations, then we say that $X$ is indecomposable. It is known that any representation $X$ can be uniquely decomposed (up to order) into a direct sum of indecomposable representations; that is, there exist indecomposable representations $X_{1}, X_{2}, \ldots, X_{s}$ such that

$$
X \cong m_{1} X_{1} \oplus m_{2} X_{2} \oplus \cdots \oplus m_{s} X_{s},
$$

where $m_{k} X_{k}=X_{k} \oplus \cdots \oplus X_{k}$ is the direct sum of $m_{k}$ copies of $X_{k}$.

In fact, if $\Gamma$ is a finite-type quiver, there are only finitely many isomorphic classes of representations of $\Gamma$, and the correspondence $X \mapsto \operatorname{dim} X$ gives a bijection between the isomorphic classes of representations and the positive roots of $\Gamma$.

Next we define homomorphisms between two representations $X$ and $Y$, where we put $\operatorname{dim} X=\left(d^{(i)}\right)_{i \in \Gamma_{0}}$ and $\operatorname{dim} Y=\left(d^{\prime(i)}\right)_{i \in \Gamma_{0}}$ respectively. A homomorphism from $X$ to $Y$ is an element $g=\left(g^{(i)}\right)_{i \in \Gamma_{0}} \in \bigoplus_{i \in \Gamma_{0}} M\left(d^{\prime(i)}, d^{(i)}\right)$ satisfying $g^{(e \alpha)} X^{(\alpha)}=Y^{(\alpha)} g^{(s \alpha)}$ for any arrow $\alpha \in \Gamma_{1}$. In other words, if we regard each matrix $X^{(\alpha)}$ as a linear map between numerical vector spaces, a homomorphism $g=\left(g^{(i)}\right)_{i \in \Gamma_{0}}$ makes the following diagram commutative for each $\alpha \in \Gamma_{1}$ :

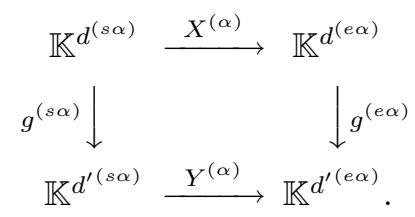

We denote by $\operatorname{Hom}(X, Y)$ the set of all homomorphisms from $X$ to $Y$, which can be regarded as a $\mathbb{K}$-vector space in the natural way.

Example 2.1. Let us consider the following $D_{4}$-type quiver $\Gamma$ :

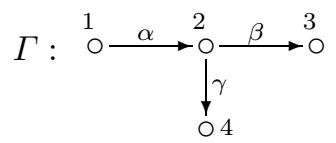

There are twelve positive roots of type $D_{4}$, which are given by the following:

$$
\begin{aligned}
& \boldsymbol{d}_{1}=(1,0,0 ; 0), \quad \boldsymbol{d}_{2}=(1,1,0 ; 0), \quad \boldsymbol{d}_{3}=(1,1,1 ; 0), \quad \boldsymbol{d}_{4}=(1,1,0 ; 1), \\
& \boldsymbol{d}_{5}=(0,1,0 ; 0), \quad \boldsymbol{d}_{6}=(1,2,1 ; 1), \quad \boldsymbol{d}_{7}=(0,1,0 ; 1), \quad \boldsymbol{d}_{8}=(0,1,1 ; 0), \\
& \boldsymbol{d}_{9}=(1,1,1 ; 1), \quad \boldsymbol{d}_{10}=(0,1,1 ; 1), \quad \boldsymbol{d}_{11}=(0,0,1 ; 0), \quad \boldsymbol{d}_{12}=(0,0,0 ; 1) .
\end{aligned}
$$

Let $X_{k}$ be an indecomposable representation corresponding to the positive root $\boldsymbol{d}_{k}$. For example, $X_{6}=\left(X^{(\alpha)}, X^{(\beta)}, X^{(\gamma)}\right) \in R_{\boldsymbol{d}_{6}}(\Gamma)=M\left(d_{6}^{(2)}, d_{6}^{(1)}\right) \oplus M\left(d_{6}^{(3)}, d_{6}^{(2)}\right) \oplus$ $M\left(d_{6}^{(4)}, d_{6}^{(2)}\right)$ is given by

$$
X^{(\alpha)}=\left[\begin{array}{l}
1 \\
0
\end{array}\right], \quad X^{(\beta)}=\left[\begin{array}{ll}
1 & 0
\end{array}\right], \quad X^{(\gamma)}=\left[\begin{array}{ll}
1 & 1
\end{array}\right] .
$$

We see that the above representative system $X_{1}, X_{2}, \ldots, X_{12}$ satisfies the condition

$$
\operatorname{Hom}\left(X_{i}, X_{j}\right)=0 \text { if } i<j .
$$

Remark 2.2. In the above example, we have numbered the positive roots of $\Gamma$ (and also a complete representative system of the isomorphic classes of its indecomposable representations) to satisfy the condition (2.1). In fact, in the case of finite-type $\Gamma$, we can always do such a numbering. Recall the so-called AuslanderReiten quiver (see, for example, Chapter VII of [2]). The vertices of the AR-quiver 
of $\Gamma$ are in one-to-one correspondence with the isomorphic classes of indecomposable representations of $\Gamma$, and there is an arrow $\left[X_{i}\right] \rightarrow\left[X_{j}\right]$ if and only if there exists an irreducible morphism $X_{i} \rightarrow X_{j}$. For example, the AR-quiver of the above $\Gamma$ is given by

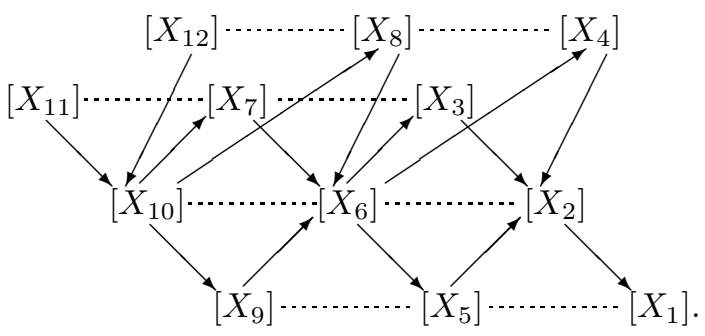

In the case where $\Gamma$ is finite-type, it is known that the AR-quiver of $\Gamma$ consists of a single component and that it is acyclic (see, for example, Proposition 5.13 of [1). Hence, if $\Gamma$ is finite-type, we may assume that a complete representative system which is numbered by an appropriate order (that is, we number from one of the tips of the component) satisfies condition (2.1).

We will denote by End $X=\operatorname{Hom}(X, X)$ the endomorphism ring of $X$ and by $H_{X}=(\text { End } X)^{\times}$its multiplicative group. In other words, $H_{X}$ is nothing but the isotropy subgroup at $X \in R_{\boldsymbol{d}}(\Gamma)$; that is, $H_{X}=\left\{g \in G_{\boldsymbol{d}} \mid g \cdot X=X\right\}$. In the case of $X \cong Y$, we see that $H_{X}$ and $H_{Y}$ are conjugate to each other.

We are interested in the restriction map $\varphi_{X}$ between rational character groups $\mathcal{X}\left(G_{\boldsymbol{d}}\right)$ and $\mathcal{X}\left(H_{X}\right)$, where we denote by $\mathcal{X}(G)$ the group consisting of all rational characters of $G$. It is known that rational character groups of linear algebraic groups are finitely generated abelian groups.

According to Proposition 1.2 of [8], the rank of $\operatorname{Im} \varphi_{X}$ describes the condition whether the $G_{\boldsymbol{d}^{-}}$orbit $G_{\boldsymbol{d}} X$ decomposes into infinitely many $S_{\boldsymbol{d}^{-}}$orbits, where we put $S_{\boldsymbol{d}}=\prod_{i \in \Gamma_{0}} S L\left(d^{(i)}\right)$. Now we note the following fact:

Lemma 2.3. Let $X$ be a point of $R_{\boldsymbol{d}}(\Gamma)$ and $\varphi_{X}: \mathcal{X}\left(G_{\boldsymbol{d}}\right) \rightarrow \mathcal{X}\left(H_{X}\right)$ the restriction map which is induced by the canonical injection $H_{X} \hookrightarrow G_{\boldsymbol{d}}$. Then, the $G_{\boldsymbol{d}^{-}}$orbit

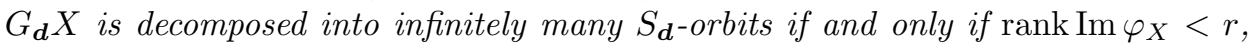
where $r$ is the number of vertices of $\Gamma$.

Proof. We put $H_{X}^{\prime}=H_{X} \cap S_{\boldsymbol{d}}$, which is a normal subgroup of $H_{X}$. Let us consider the following commutative diagram with exact rows:

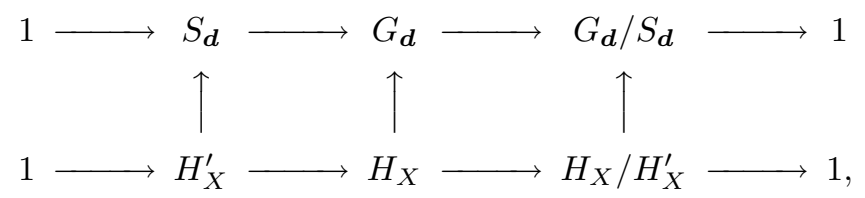


where each vertical map is the canonical injection. Then this induces the following commutative diagram with exact rows:

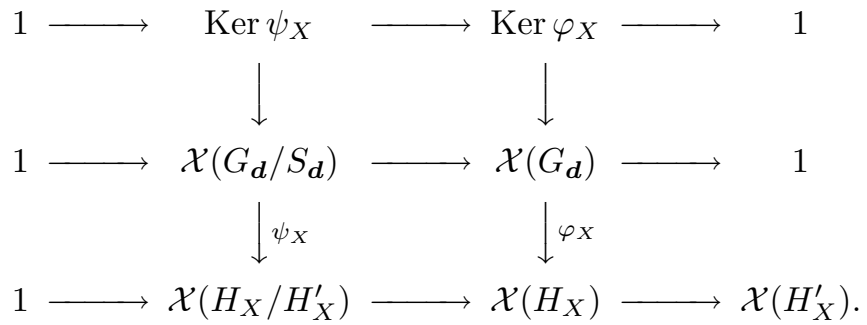

Note that $\psi_{X}$ is surjective since both $G_{\boldsymbol{d}} / S_{\boldsymbol{d}}$ and $H_{X} / H_{X}^{\prime}$ are tori. On the other hand, we see that $\mathcal{X}\left(G_{\boldsymbol{d}} / S_{\boldsymbol{d}}\right)$ is isomorphic to $\mathcal{X}\left(G_{\boldsymbol{d}}\right)$ since any character of $S_{\boldsymbol{d}}$ is trivial; hence we have $\operatorname{Ker} \psi_{X} \simeq \operatorname{Ker} \varphi_{X}$. According to Proposition 1.2 of 8 , the $G_{\boldsymbol{d}^{-}}$-orbit $G_{\boldsymbol{d}} X$ is decomposed into infinitely many $S_{\boldsymbol{d}}$-orbits if and only if $\operatorname{dim} G_{\boldsymbol{d}} X>\operatorname{dim} S_{\boldsymbol{d}} X$, which is equivalent to the condition that $r=\operatorname{rank} \mathcal{X}\left(G_{\boldsymbol{d}}\right)=$ $\operatorname{rank} \mathcal{X}\left(G_{\boldsymbol{d}} / S_{\boldsymbol{d}}\right)>\operatorname{rank} \mathcal{X}\left(H_{X} / H_{X}^{\prime}\right)$. Since these character groups are finitely generated abelian groups, we see that this condition is equivalent to the condition $r-\operatorname{rank} \operatorname{Im} \psi_{X}=\operatorname{rank} \operatorname{Ker} \psi_{X}>0$; that is, $r-\operatorname{rank} \operatorname{Im} \varphi_{X}=\operatorname{rank} \operatorname{Ker} \varphi_{X}>0$. Thus we obtain our assertion.

Lemma 2.4. Let $X$ be an indecomposable representation of a finite-type quiver $\Gamma$ and $\operatorname{dim} X=\left(d^{(i)}\right)_{i \in \Gamma_{0}}$ its dimension. Then we have

$$
\text { End } X=\left\{\left(\alpha \cdot I_{d^{(i)}}\right)_{i \in \Gamma_{0}} \mid \alpha \in \mathbb{K}\right\},
$$

where $I_{u}$ means the identity matrix of degree $u$. That is to say, End $X$ is isomorphic to the base field $\mathbb{K}$ and each component is a scalar matrix.

Proof. Put $T=\left\{\left(\alpha \cdot I_{d^{(i)}}\right)_{i \in \Gamma_{0}} \mid \alpha \in \mathbb{K}\right\}$; then $T$ is a field that is isomorphic to the field $\mathbb{K}$. It is clear that $T$ is contained in End $X$, which can be regarded as a $\mathbb{K}$-vector space. On the other hand, it is known that, for each indecomposable representation $X$ of a finite-type quiver, End $X$ is nothing but the base field $\mathbb{K}$ (see [3], $\S 7.2$ ). Since End $X$ is finite dimensional over the field $T$, we have End $X=T$.

Lemma 2.5. Let $X$ be an indecomposable representation, with dimension $\operatorname{dim} X=$ $\left(d^{(i)}\right)_{i \in \Gamma_{0}}$, of a finite-type quiver. For each positive integer $m$, the endomorphism ring $\operatorname{End}(m X)$ of $m X=X \oplus \cdots \oplus X$ (the direct sum of $m$ copies of $X)$ is given by

$$
\operatorname{End}(m X)=\left\{\left(A \otimes I_{d^{(i)}}\right)_{i \in \Gamma_{0}} \mid A \in M(m, m)\right\} \simeq M(m, m)
$$

where $\otimes$ denotes Kronecker's product of matrices. In particular, we have $H_{m X} \simeq$ $G L(m)$ and the rational character group $\mathcal{X}\left(H_{m X}\right)$ is of rank one.

Proof. Let $g=\left(g^{(i)}\right)_{i \in \Gamma_{0}}$ be an element of $\operatorname{End}(m X)$, and write each part $g^{(i)}$ as the following $m \times m$ blocks:

$$
g^{(i)}=\left[\begin{array}{ccc}
g_{11}^{(i)} & \cdots & g_{1 m}^{(i)} \\
\vdots & \ddots & \vdots \\
g_{m 1}^{(i)} & \cdots & g_{m m}^{(i)}
\end{array}\right],
$$

where each block $g_{p q}^{(i)}$ is a $d^{(i)} \times d^{(i)}$ matrix. Then, for each arrow $\alpha \in \Gamma_{1}$, we have

$$
g_{p q}^{(e \alpha)} X^{(\alpha)}=X^{(\alpha)} g_{p q}^{(s \alpha)} \quad(p, q=1,2, \ldots, m) .
$$


That is to say, for each $p$ and $q$, we see that $\left(g_{p q}^{(i)}\right)_{i \in \Gamma_{0}}$ is contained in the endomorphism ring End $X$ of an indecomposable $X$. Therefore it follows from Lemma 2.4 that, for each $p$ and $q$, there exists a scalar $\alpha_{p q} \in \mathbb{K}$ satisfying $g_{p q}^{(i)}=\alpha_{p q} \cdot I_{d^{(i)}}$. Putting $A=\left[\alpha_{p q}\right] \in M(m, m)$, we have $g^{(i)}=A \otimes I_{d^{(i)}}$.

Proposition 2.6. Let $X=m_{1} X_{1} \oplus m_{2} X_{2} \oplus \cdots \oplus m_{s} X_{s} \in R_{\boldsymbol{d}}(\Gamma)$ be a representation, where the $X_{i}$ 's are distinct indecomposable representations which are numbered to satisfy condition (2.1). Then we have $\operatorname{rank} \mathcal{X}\left(H_{X}\right)=s$.

Proof. Put $\operatorname{dim} X_{k}=\boldsymbol{d}_{k}=\left(d_{k}^{(i)}\right)_{i \in \Gamma_{0}}$, and let $\tilde{X}_{k}=m_{k} X_{k}$ be the direct sum of $m_{k}$ copies of $X_{k}$. Then we have $\operatorname{dim} \tilde{X}_{k}=m_{k} \boldsymbol{d}_{k}=\left(m_{k} d_{k}^{(i)}\right)_{i \in \Gamma_{0}}$. Let $h=\left(h^{(i)}\right)_{i \in \Gamma_{0}}$ be an element of the isotropy subgroup $H_{\tilde{X}_{1} \oplus \cdots \oplus \tilde{X}_{s}}$, and decompose each part $h^{(i)}$ into $s \times s$ blocks:

$$
h^{(i)}=\left[\begin{array}{ccc}
h_{11}^{(i)} & \cdots & h_{1 s}^{(i)} \\
\vdots & \ddots & \vdots \\
h_{s 1}^{(i)} & \cdots & h_{s s}^{(i)}
\end{array}\right],
$$

where each block $h_{p q}^{(i)}$ is an $m_{p} d_{p}^{(i)} \times m_{q} d_{q}^{(i)}$ matrix. In the case of $d_{k}^{(i)}=0$, we should remove its corresponding blocks. Thus we have

$$
h_{p q}^{(e \alpha)} \tilde{X}_{q}^{(\alpha)}=\tilde{X}_{p}^{(\alpha)} h_{p q}^{(s \alpha)}
$$

for each arrow $\alpha \in \Gamma_{1}$, and hence $h_{p q}=\left(h_{p q}^{(i)}\right)_{i \in \Gamma_{0}} \in \operatorname{Hom}\left(\tilde{X}_{q}, \tilde{X}_{p}\right)$. Then condition (2.1) implies $h_{p q}=\left(h_{p q}^{(i)}\right)_{i \in \Gamma_{0}}=0$ for any $p$ and $q$ satisfying $q<p$. Therefore we see that each part $h^{(i)}$ is contained in a subgroup consisting of upper triangular block matrices (i.e., it is contained in the standard parabolic subgroup corresponding to the partition $\left.m_{1} d_{1}^{(i)}+m_{2} d_{2}^{(i)}+\cdots+m_{s} d_{s}^{(i)}\right)$ :

$$
h^{(i)}=\left[\begin{array}{cccc}
h_{11}^{(i)} & h_{12}^{(i)} & \ldots & h_{1 s}^{(i)} \\
0 & h_{22}^{(i)} & \ldots & h_{2 s}^{(i)} \\
\vdots & \ddots & \ddots & \vdots \\
0 & \ldots & 0 & h_{s s}^{(i)}
\end{array}\right] .
$$

Hence, for $k=1,2, \ldots, s$, we have the canonical projection $H_{X} \rightarrow H_{\tilde{X}_{k}}$ by $h=$ $\left(h^{(i)}\right)_{i \in \Gamma_{0}} \mapsto\left(h_{k k}^{(i)}\right)_{i \in \Gamma_{0}}$ (here we will consider $h_{k k}^{(i)}$ to be trivial if $d_{k}^{(i)}=0$ ). It follows from Lemma 2.5 that there exists $A_{k} \in G L\left(m_{k}\right)$ satisfying $h_{k k}^{(i)}=A_{k} \otimes I_{d_{k}^{(i)}}$ for any $i \in \Gamma_{0}$; hence we can define the character $\lambda_{k}(h)=\operatorname{det} A_{k}$ for $h \in H_{X}$. Then we see that each rational character group $\mathcal{X}\left(H_{\tilde{X}_{k}}\right)$ is generated by $\lambda_{k}$ and that $\lambda_{1}, \lambda_{2}, \ldots, \lambda_{s}$ constitute a basis of the rational character group $\mathcal{X}\left(H_{X}\right)$; that is, it is a free abelian group of rank $s$.

For each $i \in \Gamma_{0}$, we define the character $\chi_{i}: G_{\boldsymbol{d}} \rightarrow \mathbb{K}^{\times}$by $\chi_{i}(g)=\operatorname{det} g^{(i)}$ for $g=\left(g^{(i)}\right)_{i \in \Gamma_{0}} \in G_{\boldsymbol{d}}$.

Corollary 2.7. In the same notation as in Proposition 2.6, the representation matrix of the restriction map $\varphi_{X}: \mathcal{X}\left(G_{\boldsymbol{d}}\right) \rightarrow \mathcal{X}\left(H_{X}\right)$, with respect to bases $\chi_{1}, \chi_{2}$, $\ldots, \chi_{r}$ and $\lambda_{1}, \lambda_{2}, \ldots, \lambda_{s}$, is given by $\left[{ }^{t} \boldsymbol{d}_{1}\left|{ }^{t} \boldsymbol{d}_{2}\right| \cdots \mid{ }^{t} \boldsymbol{d}_{s}\right]$, where $\boldsymbol{d}_{k}$ is the positive root corresponding to $X_{k}$. 
Proof. Take an element $h=\left(h^{(i)}\right)_{i \in \Gamma_{0}} \in H_{X}$. Then we have

$$
\begin{aligned}
\chi_{i}(h)=\operatorname{det}\left(h_{11}^{(i)} h_{22}^{(i)} \cdots h_{s s}^{(i)}\right) & =\left(\operatorname{det} h_{11}^{(i)}\right)\left(\operatorname{det} h_{22}^{(i)}\right) \cdots\left(\operatorname{det} h_{s s}^{(i)}\right) \\
& =\left(\lambda_{1}(h)\right)^{d_{1}^{(i)}}\left(\lambda_{2}(h)\right)^{d_{2}^{(i)}} \cdots\left(\lambda_{s}(h)\right)^{d_{s}^{(i)}} \\
& =\left(\lambda_{1}^{d_{1}^{(i)}} \lambda_{2}^{d_{2}^{(i)}} \cdots \lambda_{s}^{d_{s}^{(i)}}\right)(h)
\end{aligned}
$$

for each $i \in \Gamma_{0}$. Hence the representation matrix of $\varphi_{X}$ with respect to such bases is given by

$$
\left[\begin{array}{cccc}
d_{1}^{(1)} & d_{2}^{(1)} & \cdots & d_{s}^{(1)} \\
d_{1}^{(2)} & d_{2}^{(2)} & \cdots & d_{s}^{(2)} \\
\vdots & \vdots & & \vdots \\
d_{1}^{(r)} & d_{2}^{(r)} & \cdots & d_{s}^{(r)}
\end{array}\right]=\left[\left.{ }^{t} \boldsymbol{d}_{1}\right|^{t} \boldsymbol{d}_{2}|\cdots|{ }^{t} \boldsymbol{d}_{s}\right]
$$

that is, for each $k$, the $k$-th column is nothing but the transpose of the positive root $\boldsymbol{d}_{k}$.

\section{Characterization of Semisimple FPs}

Now we are standing at the position required to prove our main theorem:

Theorem 3.1. Let $\Gamma$ be a finite-type quiver with $r$ vertices. For a dimension vector $\boldsymbol{d}$, the following conditions are equivalent:

(1) The scalar-removed representation $\left(S_{\boldsymbol{d}}, R_{\boldsymbol{d}}(\Gamma)\right)$ is not an $F P$.

(2) There exist some positive roots $\boldsymbol{d}_{i_{1}}, \boldsymbol{d}_{i_{2}}, \ldots, \boldsymbol{d}_{i_{p}}$ of $\Gamma$ such that $\boldsymbol{d} \in\left\langle\boldsymbol{d}_{i_{1}}, \boldsymbol{d}_{i_{2}}\right.$, $\left.\ldots, \boldsymbol{d}_{i_{p}}\right\rangle_{\mathbb{Z}_{\geq 0}}$ and $\operatorname{rank}\left[\left.{ }^{t} \boldsymbol{d}_{i_{1}}\right|^{t} \boldsymbol{d}_{i_{2}}|\cdots|{ }^{t} \boldsymbol{d}_{i_{p}}\right]<r$.

Proof. Assume that $\left(S_{\boldsymbol{d}}, R_{\boldsymbol{d}}(\Gamma)\right)$ is not an FP. Then there exists a point $X \in$ $R_{\boldsymbol{d}}(\Gamma)$ such that its $G_{\boldsymbol{d}^{\text {-orbit }}}$ is decomposed into infinitely many $S_{\boldsymbol{d}}$-orbits. By Lemma 2.3. this is equivalent to the condition that $\operatorname{rank} \operatorname{Im} \varphi_{X}<r$. Now we can choose some positive integers $m_{1}, m_{2}, \ldots, m_{p}$ and indecomposable representations $X_{i_{1}}, X_{i_{2}}, \ldots, X_{i_{p}}$ such that $X \cong m_{1} X_{i_{1}} \oplus m_{2} X_{i_{2}} \oplus \cdots \oplus m_{p} X_{i_{p}}$. Here, as mentioned in Remark 2.2, we may assume that the $X_{k}$ 's are numbered to satisfy the condition (2.1). Then it follows from Corollary 2.7 that $\operatorname{rank} \operatorname{Im} \varphi_{X}=\operatorname{rank}\left[{ }^{t} \boldsymbol{d}_{i_{1}}\left|{ }^{t} \boldsymbol{d}_{i_{2}}\right| \cdots \mid{ }^{t} \boldsymbol{d}_{i_{p}}\right]$, and we have $\boldsymbol{d}=\operatorname{dim} X=m_{1} \boldsymbol{d}_{i_{1}}+m_{2} \boldsymbol{d}_{i_{2}}+\cdots+m_{p} \boldsymbol{d}_{i_{p}}$; therefore we obtain (2).

Conversely, the condition $\boldsymbol{d} \in\left\langle\boldsymbol{d}_{i_{1}}, \boldsymbol{d}_{i_{2}}, \ldots, \boldsymbol{d}_{i_{p}}\right\rangle_{\mathbb{Z}_{>0}}$ implies that we can construct the representation $X=m_{1} X_{i_{1}} \oplus m_{2} X_{i_{2}} \oplus \cdots \oplus m_{p} X_{i_{p}} \in R_{\boldsymbol{d}}(\Gamma)$. Then the second

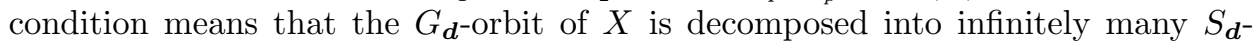
orbits; i.e., the representation $\left(S_{\boldsymbol{d}}, R_{\boldsymbol{d}}(\Gamma)\right)$ is not an FP.

In fact, condition (2) of Theorem 3.1 can be improved a little. Here we will review a few properties of positive roots.

Let $E$ be a Euclidean space (over $\mathbb{R}$ ) endowed with an appropriate inner product. Fix a basis of $E$ and define the lexicographical order with respect to the basis. Let $\Phi^{+}$be the set of all positive roots contained in a root system of $E$.

For a finite subset $M \subseteq \Phi^{+}$we put $\Psi=\langle M\rangle_{\mathbb{R}} \cap \Phi^{+}$and $\operatorname{dim}_{\mathbb{R}}\langle M\rangle_{\mathbb{R}}=p$; i.e., the subspace generated by $M$ is of dimension $p$. Now we choose $p$ positive roots $\boldsymbol{\alpha}_{1}, \boldsymbol{\alpha}_{2}, \ldots, \boldsymbol{\alpha}_{p}$ as follows:

$$
\boldsymbol{\alpha}_{1}:=\min \Psi, \text { and } \boldsymbol{\alpha}_{k}:=\min \left(\Psi \backslash\left\langle\boldsymbol{\alpha}_{1}, \ldots, \boldsymbol{\alpha}_{k-1}\right\rangle_{\mathbb{R}}\right) \text { for } k=2,3, \ldots, p .
$$


Then we have the following lemma, which can be proved by induction on the dimension of $\langle M\rangle_{\mathbb{R}}$.

Lemma 3.2. For any element $\boldsymbol{\alpha} \in \Psi$, there exist non-negative integers $k_{1}, k_{2}, \ldots$, $k_{p}$ such that $\boldsymbol{\alpha}=k_{1} \boldsymbol{\alpha}_{1}+k_{2} \boldsymbol{\alpha}_{2}+\cdots+k_{p} \boldsymbol{\alpha}_{p}$.

Proposition 3.3. Let $\boldsymbol{d}_{1}, \boldsymbol{d}_{2}, \ldots, \boldsymbol{d}_{s}$ be positive roots contained in a root system of a Euclidean space. If $\operatorname{rank}_{\mathbb{Z}}\left\langle\boldsymbol{d}_{1}, \boldsymbol{d}_{2}, \ldots, \boldsymbol{d}_{s}\right\rangle_{\mathbb{Z}}=p$, then there exist $p$ positive roots $\boldsymbol{\alpha}_{1}, \boldsymbol{\alpha}_{2}, \ldots, \boldsymbol{\alpha}_{p}$ such that $\left\langle\boldsymbol{d}_{1}, \boldsymbol{d}_{2}, \ldots, \boldsymbol{d}_{s}\right\rangle_{\mathbb{Z}}=\left\langle\boldsymbol{\alpha}_{1}, \boldsymbol{\alpha}_{2}, \ldots, \boldsymbol{\alpha}_{p}\right\rangle_{\mathbb{Z}}$. In particular, we have $\left\langle\boldsymbol{d}_{1}, \boldsymbol{d}_{2}, \ldots, \boldsymbol{d}_{s}\right\rangle_{\mathbb{Z}_{>0}} \subseteq\left\langle\boldsymbol{\alpha}_{1}, \boldsymbol{\alpha}_{2}, \ldots, \boldsymbol{\alpha}_{p}\right\rangle_{\mathbb{Z}_{>0}}$; that is, the lattice with coefficients of non-negative integers spanned by $\boldsymbol{d}_{1}, \boldsymbol{d}_{2}, \ldots, \boldsymbol{d}_{s}$ is contained in one spanned by $\boldsymbol{\alpha}_{1}, \boldsymbol{\alpha}_{2}, \ldots, \boldsymbol{\alpha}_{p}$.

Proof. Put $M:=\left\{\boldsymbol{d}_{1}, \boldsymbol{d}_{2}, \ldots, \boldsymbol{d}_{s}\right\}$. Since $\langle M\rangle_{\mathbb{R}}=\mathbb{R} \otimes_{\mathbb{Z}}\left\langle\boldsymbol{d}_{1}, \boldsymbol{d}_{2}, \ldots, \boldsymbol{d}_{s}\right\rangle_{\mathbb{Z}}$, we have $\operatorname{dim}_{\mathbb{R}}\langle M\rangle_{\mathbb{R}}=\operatorname{rank}_{\mathbb{Z}}\left\langle\boldsymbol{d}_{1}, \boldsymbol{d}_{2}, \ldots, \boldsymbol{d}_{s}\right\rangle_{\mathbb{Z}}=p$. By Lemma 3.2, each $\boldsymbol{d}_{k}$ can be expressed as a linear combination of $\boldsymbol{\alpha}_{1}, \boldsymbol{\alpha}_{2}, \ldots, \boldsymbol{\alpha}_{p}$ with coefficients of non-negative integers. Thus we obtain our assertion.

Therefore we have gained a more sophisticated characterization of scalar-removed FPs associated with finite-type quivers.

Theorem 3.4. Let $\Gamma$ be a finite-type quiver with $r$ vertices. For a dimension vector $\boldsymbol{d}$, the following conditions are equivalent:

(1) The scalar-removed representation $\left(S_{\boldsymbol{d}}, R_{\boldsymbol{d}}(\Gamma)\right)$ is not an FP.

(2) There exist $r-1$ positive roots $\boldsymbol{d}_{i_{1}}, \boldsymbol{d}_{i_{2}}, \ldots, \boldsymbol{d}_{i_{r-1}}$ of $\Gamma$ satisfying $\boldsymbol{d} \in\left\langle\boldsymbol{d}_{i_{1}}\right.$, $\left.\boldsymbol{d}_{i_{2}}, \ldots, \boldsymbol{d}_{i_{r-1}}\right\rangle_{\mathbb{Z}_{\geq 0}}$; that is, $\boldsymbol{d}$ can be expressed as a linear combination of $r-1$ positive roots with coefficients of non-negative integers.

In particular, the condition whether $\left(S_{\boldsymbol{d}}, R_{\boldsymbol{d}}(\Gamma)\right)$ is an FP or not does not depend on the choice of an orientation of $\Gamma$.

\section{Examples of $D_{4}$-TYPE}

In this section, we give some examples of $D_{4}$-type. Let $\Gamma$ be the $D_{4}$-type quiver mentioned in Example 2.1. We are interested in lattices of small rank because we will determine dimension $\boldsymbol{d}$ such that $\left(S_{\boldsymbol{d}}, R_{\boldsymbol{d}}(\Gamma)\right)$ is not an FP.

First we note that there exist twenty distinct lattices of rank three (with each component of the sum of generators being positive) spanned by positive roots of $D_{4}$-type.
(1) $L_{1}=\langle 2,11,12\rangle$
(8) $L_{8}=\langle 1,7,8\rangle$
(15) $L_{15}=\langle 1,6,11\rangle$
(2) $L_{2}=\langle 1,8,12\rangle$
(9) $L_{9}=\langle 2,7,11\rangle$
(16) $L_{16}=\langle 2,8,9\rangle$
(3) $L_{3}=\langle 1,7,11\rangle$
(10) $L_{10}=\langle 4,5,10\rangle$
(17) $L_{17}=\langle 7,8,9\rangle$
(4) $L_{4}=\langle 3,5,12\rangle$
(11) $L_{11}=\langle 3,4,5\rangle$
(18) $L_{18}=\langle 2,7,9\rangle$
(5) $L_{5}=\langle 1,5,10\rangle$
(12) $L_{12}=\langle 3,5,10\rangle$
(19) $L_{19}=\langle 2,7,8\rangle$
(6) $L_{6}=\langle 4,5,11\rangle$
(13) $L_{13}=\langle 6,11,12\rangle$
(20) $L_{20}=\langle 3,4,10\rangle$
(7) $L_{7}=\langle 2,8,12\rangle$
(14) $L_{14}=\langle 1,6,12\rangle$

In the above list, for example, $L_{1}=\langle 2,11,12\rangle$ means that the lattice (free $\mathbb{Z}$-module) $L_{1}$ is spanned by three roots $\boldsymbol{d}_{2}, \boldsymbol{d}_{11}, \boldsymbol{d}_{12}$ (we recall that the roots of $D_{4}$-type $\Gamma$ have been numbered in Example 2.1). Therefore a dimension vector (i.e., a four-tuple of positive integers) $\boldsymbol{d}=\left(d^{(1)}, d^{(2)}, d^{(3)} ; d^{(4)}\right)$ is contained in $L_{1}$ if and only if $d^{(1)}=d^{(2)}$. Thus we obtain the following theorem for $D_{4}$-type: 
Theorem 4.1. Let $\Gamma$ be a $D_{4}$-type quiver. Then, for a given dimension vector $\boldsymbol{d}=\left(d^{(1)}, d^{(2)}, d^{(3)} ; d^{(4)}\right)$, the scalar-removed representation $\left(S_{\boldsymbol{d}}, R_{\boldsymbol{d}}(\Gamma)\right)$ is not an $F P$ if and only if at least one of the following twenty conditions is satisfied:
(1) $d^{(1)}=d^{(2)}$
(2) $d^{(3)}=d^{(2)}$
(3) $d^{(4)}=d^{(2)}$
(4) $d^{(1)}=d^{(3)}<d^{(2)}$
(5) $d^{(3)}=d^{(4)}<d^{(2)}$
(6) $d^{(4)}=d^{(1)}<d^{(2)}$
(7) $d^{(1)}+d^{(3)}=d^{(2)}$
(8) $d^{(3)}+d^{(4)}=d^{(2)}$
(9) $d^{(4)}+d^{(1)}=d^{(2)}$
(10) $d^{(1)}+d^{(3)}=d^{(4)}<d^{(2)}$
(11) $d^{(3)}+d^{(4)}=d^{(1)}<d^{(2)}$
(12) $d^{(4)}+d^{(1)}=d^{(3)}<d^{(2)}$
(13) $2 d^{(1)}=d^{(2)}$
and $d^{(1)}<\min \left\{d^{(3)}, d^{(4)}\right\}$
(14) $2 d^{(3)}=d^{(2)}$
and $d^{(3)}<\min \left\{d^{(4)}, d^{(1)}\right\}$
(15) $2 d^{(4)}=d^{(2)}$
and $d^{(4)}<\min \left\{d^{(1)}, d^{(3)}\right\}$
(16) $d^{(1)}+d^{(3)}=d^{(4)}+d^{(2)}$
and $\max \left\{d^{(1)}, d^{(3)}\right\}<d^{(2)}$
(17) $d^{(3)}+d^{(4)}=d^{(1)}+d^{(2)}$
and $\max \left\{d^{(3)}, d^{(4)}\right\}<d^{(2)}$
(18) $d^{(4)}+d^{(1)}=d^{(3)}+d^{(2)}$
and $\max \left\{d^{(4)}, d^{(1)}\right\}<d^{(2)}$
(19) $d^{(1)}+d^{(3)}+d^{(4)}=d^{(2)}$
(20) $d^{(1)}+d^{(3)}+d^{(4)}=2 d^{(2)}$
and $\max \left\{d^{(1)}, d^{(3)}, d^{(4)}\right\}<d^{(2)}$

Note that Theorem 4.1 was independently obtained by Dr. Tomohiro Kamiyoshi, a researcher (non-full-time) at the University of Tsukuba. He has investigated representations associated with $D_{4}$-type quivers under various scalar restrictions (see [4]).

Among $D_{4}$-type FPs $\left(S_{\boldsymbol{d}}, R_{\boldsymbol{d}}(\Gamma)\right)$, we are interested in representations of dimension $\boldsymbol{d}=\left(d^{(1)}, d^{(2)}, d^{(3)} ; d^{(4)}\right)$ satisfying $d^{(2)}>\max \left\{d^{(1)}, d^{(3)}, d^{(4)}\right\}$, because if an $A_{3}$-type representation of dimension $\left(d^{(1)}, d^{(2)}, d^{(3)}\right)$ is an $\mathrm{FP}$, then so is any $D_{4}$-type with dimensional condition $d^{(2)}<d^{(4)}$. (Recall the elementary transformations of matrices. A precise statement is mentioned in, for example, [5, Proposition 1.3].)

Example 4.2. For $\boldsymbol{d}=(2,8,3 ; 4)$, we have $\boldsymbol{d}=-2 \boldsymbol{d}_{1}+4 \boldsymbol{d}_{6}-\boldsymbol{d}_{11}$ and hence $\boldsymbol{d} \in L_{15}$. However, we can conclude that $\left(S_{\boldsymbol{d}}, R_{\boldsymbol{d}}(\Gamma)\right)$ is an FP, because $\boldsymbol{d}$ cannot be expressed as a linear combination of positive roots with coefficients of non-negative integers (i.e., the dimension $\boldsymbol{d}$ does not satisfy any of the twenty conditions listed in Theorem 4.1). In fact, $R_{\boldsymbol{d}}(\Gamma)$ is decomposed into $439 S_{\boldsymbol{d}^{-}}$orbits.

Thus we realize that the conditions on $\boldsymbol{d}$ whether $\left(S_{\boldsymbol{d}}, R_{\boldsymbol{d}}(\Gamma)\right)$ is an FP or not can be obtained in this way. To know such conditions, it is sufficient to list lattices of small rank. For example, there exist 26 (resp. 76, 633) lattices of $A_{5}$-type (resp. $D_{5}, E_{6}$-type) of small rank with each component of the sum of generators being positive.

\section{ACKNOWLEDGMENTS}

The authors must give thanks to Dr. Hiroshi Nagase, an assistant professor at Nara National College of Technology, who pointed out to the first author how ARsequences are effective in the calculation of isotropy subgroups. The authors also thank Professor Dr. Tatsuo Kimura at the University of Tsukuba, who introduced the authors to this interesting open problem: "try to classify all semisimple finite prehomogeneous vector spaces". 


\section{REFERENCES}

[1] I. Assem, D. Simson, and A. Skowroński, Elements of the representation theory of associative algebras. Vol. 1, London Mathematical Society Student Texts 65, Cambridge University Press, 2006. MR2197389(2006j:16020)

[2] M. Auslander, I. Reiten, and S. O. Smalø, Representation theory of Artin algebras, Cambridge Studies in Advanced Mathematics 36, Cambridge University Press, 1997. MR 1476671 (98e:16011)

[3] P. Gabriel and A. V. Roiter, Representations of finite-dimensional algebras, Springer-Verlag, 1997. MR1475926 (98e:16014)

[4] T. Kamiyoshi, A characterization of finite prehomogeneous vector spaces of $D_{4}$-type under various scalar restrictions, submitted to Tsukuba J. of Math.

[5] T. Kimura, T. Kamiyoshi, N. Maki, M. Ouchi, and M. Takano, A classification of reductive finite prehomogeneous vector spaces of type $\left(G \times G L_{n}, \rho \otimes \Lambda_{1}\right)(n \geq 2)$ under various restricted scalar multiplications, preprint.

[6] T. Kimura, S. Kasai, and O. Yasukura, A classification of the representations of reductive algebraic groups which admit only a finite number of orbits, Amer. J. Math. 108 (1986), no. 3, 643-691. MR844634 (87k:20074)

[7] M. Nagura and T. Niitani, Conditions on a finite number of orbits for $A_{r}$-type quivers, J. Algebra 274 (2004), no. 2, 429-445. MR2043357 (2004m:16021)

[8] M. Nagura, T. Niitani, and S. Otani, A remark on prehomogeneous actions of linear algebraic groups, Nihonkai Math. J. 14 (2003), no. 2, 113-119. MR2028468 (2004m:20088)

[9] M. Sato and T. Kimura, A classification of irreducible prehomogeneous vector spaces and their relative invariants, Nagoya Math. J. 65 (1977), 1-155. MR0430336 (55:3341)

Department of Liberal Studies, Nara National College of Technology, YamatoKoriyama, NARA 639-1080, JAPAN

E-mail address: nagura@libe.nara-k.ac.jp

School of Engineering, Kanto-Gakuin University, Yokohama, Kanagawa 236-8501, JAPAN

E-mail address: hocke@kanto-gakuin.ac.jp

Castle Tsuchiura 205, Fujisaki 1-4-6, Tsuchiura, Ibaraki 300-0813, Japan

E-mail address: d-takeda@f3.dion.ne.jp 